\title{
Effect of Eight Weeks of Modified Pilates Training on Pulmonary Function and Quality of Life of Veterans Exposed to Chemical Warfare Agents
}

\section{ART ICLE INF O}

\section{Article Type}

Original Research

\section{Authors}

Ghasemi Gh. ${ }^{* 1} P h D$,

Barati Rokati P. ${ }^{2} M S c$,

Salehi O.R. ${ }^{3} M S C$

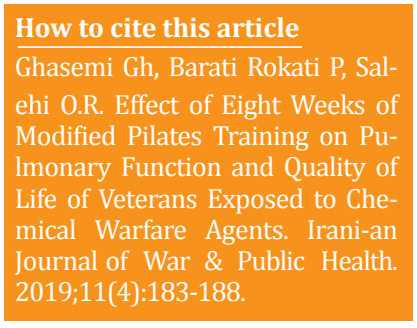

\section{A B S T R A C T}

Aim(s) Injury with chemical agents effects on the physical, mental, social and spiritual dimension of quality of life. The aim of the present study was to investigate the effect of eight weeks of modified Pilates training on pulmonary function and quality of life in veterans exposed to chemical warfare agents.

Materials and Methods This semi-experimental study with pretest-posttest design and a control group was conducted in Izeh County in 2016. The study was carried out on 28 male veterans who were exposed to chemical warfare agents. The subjects were selected by convenience sampling method and randomly assigned into the control group $(n=15)$ and training group $(n=13)$. The training group performed the modified Pilates training for 8 weeks, 3 sessions-in 60 minutes per week. The control group did not participate in any particular training program during this period. Muscular strength, pulmonary index, and quality of life of the subjects before and after the training period were measured by hand dynamometer, spirometer, and quality of life questionnaire respectively. Data were analyzed through SPSS 22 software using paired sample t-test and univariate analysis of covariance test.

Findings The mean scores of forced vital capacity and the quality of life in terms of mental and physical dimensions in the posttest step were significantly higher than the pretest step $(p=0.001)$. By removing the pretest effect, the mean scores of back muscular power $(p=0.03)$, forced vital capacity $(p=0.02)$ and quality of life $(p=0.001)$ in the training group were significantly higher than the control group.

Conclusion Eight weeks of modified Pilates training can be effective in the improvement of pulmonary function and quality of life of veterans exposed to chemical warfare agents.

Keywords Pilates Training; Chemical Warfare; Veteran; Pulmonary Function; Quality of Life
${ }^{1}$ Sport Injuries \& Corrective Exercises Department, Sport Sciences Faculty, University of Isfahan, Isfahan, Iran

${ }^{2}$ Physical Education\& Sport Sciences Department, Physical Education\& Sport Sciences Faculty, Isfahan(Khorasgan) Branch, Islamic Azad University, Isfahan, Iran ${ }^{3}$ Physical Education \& Sport Sciences Department, Humanities \& Social Sciences Faculty, University of Kurdestan, Sanandaj, Iran

\section{*Correspondence}

Address: Sport Sciences Faculty, University of Isfahan, Azadi Square, Isfahan, Iran. Postal Code: 8174673441 Phone: +98 (31) 37932571

Fax: +98 (31) 37932572

gh.ghasemi@spr.ui.ac.ir

\section{Article History}

Received: November 24, 2018

Accepted: June 17, 2019

ePublished: December 21, 2019

\section{I T A T I O N L INKS}

[1] Serum level of SDF-1 $\alpha$ (CXCL12) in chemical victims with respiratory ... [2] Effect of a training program on quality of life of severe respiratory chemical ... [3] The short-term effect of chest physiotherapy on spirometric indices in chemical ... [4] A review on the delayed complications of sulphur mustard ... [5] Increased levels of vascular endothelial growth factor in induced sputum in ... [6] Influence of structural corrective and respiratory exercises on cardiorespiratory ... [7] The effect of pulmonary rehabilitation program on quality of life of elderly patients ... [8] The effects of modified Pilates training on quality of life and clinical symptoms in ... [9] The effect of a selected aerobic exercise course on the rate of FEV1 and FVC, activity ... [10] The effects of aerobic and strength exercises on pulmonary function tests ... [11] The Pilates method increases respiratory muscle ... [12] The influence of inspiratory muscle training combined with the Pilates ... [13] Pilates-based therapeutic exercise: effect on subjects with nonspecific chronic ... [14] Pilates and the stroke ... [15] Quality of life in chemical war victims with sever ... [16] Studying the surrogate validity of respiratory indexes in predicting the ... [17] The effect of breathing exercises on fatigue level of COPD ... [18] The effect of 8 weeks specific aquatic therapy on kyphosis angle and some pulmonary ... [19] The effect of 8 weeks yoga training on respiratory function and heart of ... [20] The effects of eight weeks of resistance training on some muscle hypertrophy and ... [21] Effect of six week Pilates exercises on disability, abdominal and back ... [22] The effect of selected Pilates exercises on thigh muscle strength and ... [23] The effects of selected Pilates exercises on muscle strength ... [24] Quality of life of chemically-disabled war veterans involved ... [25] Effect of 12 weeks of chosen Pilates exercise ... 


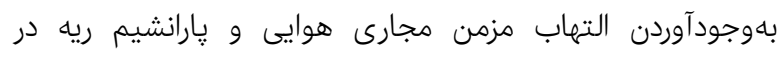

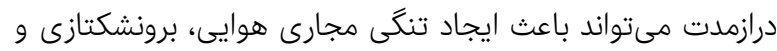

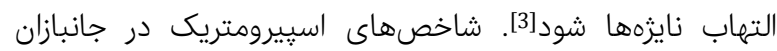

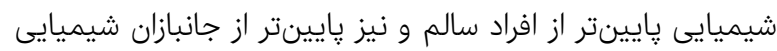

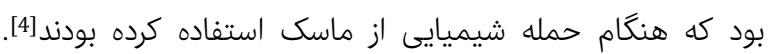

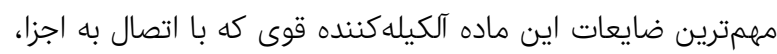

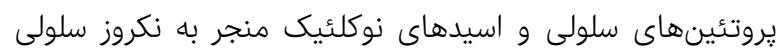

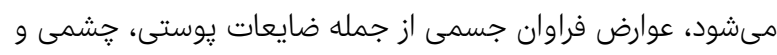

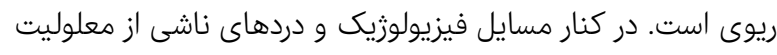

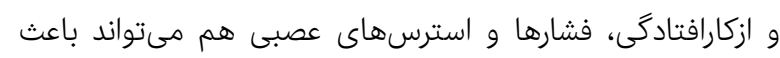

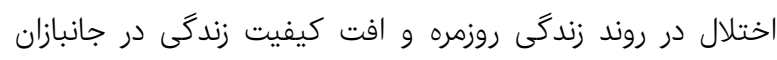

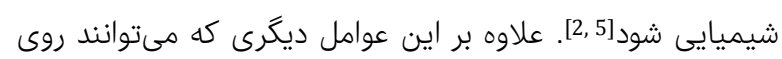

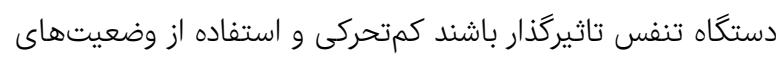

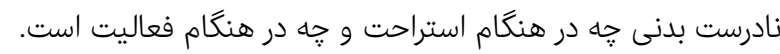

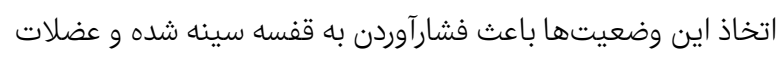

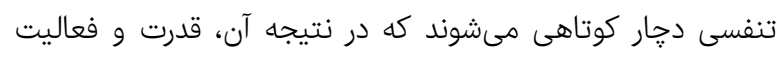

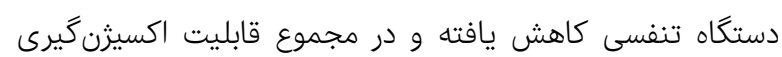

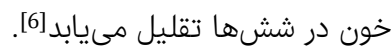

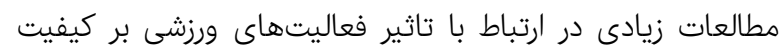

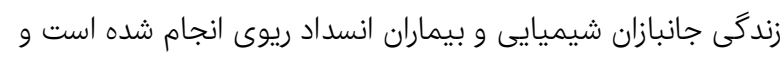

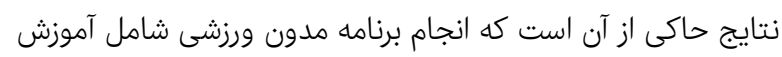

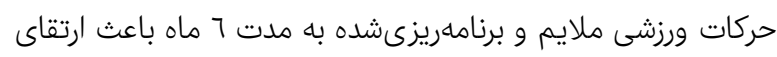

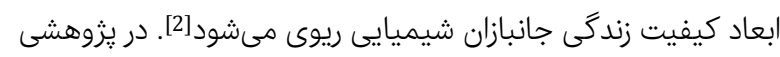

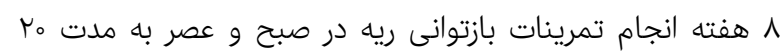

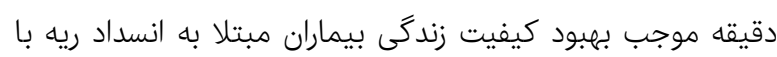

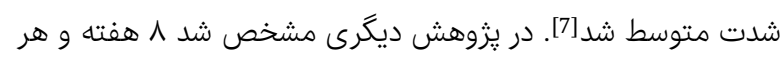

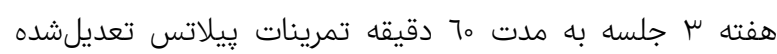

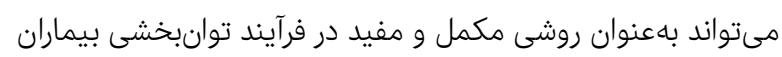

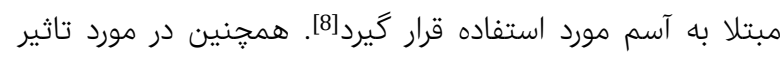

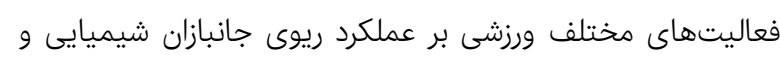

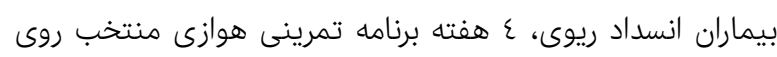

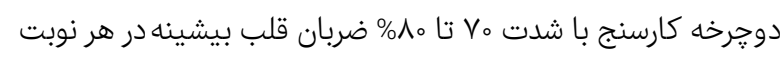

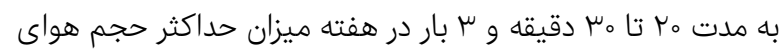

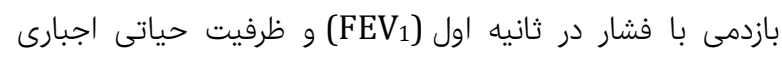

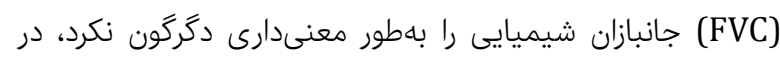

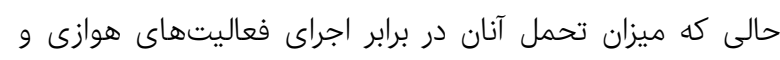

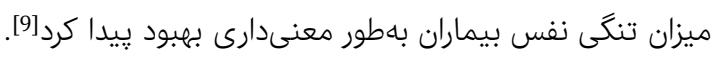

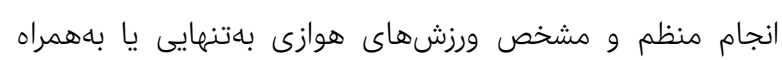

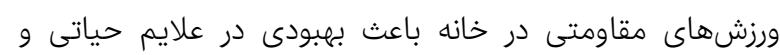

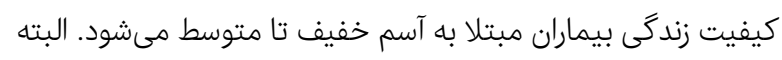

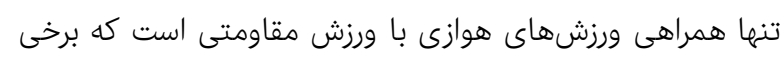

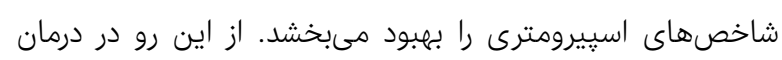

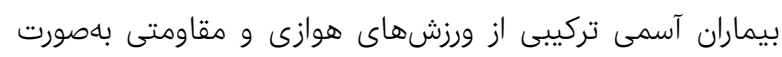

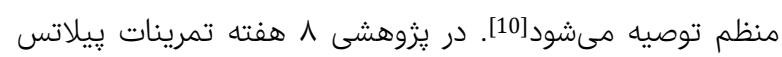

\section{اثر هشت هفته تمرين پييلاتس تعديلشده بران

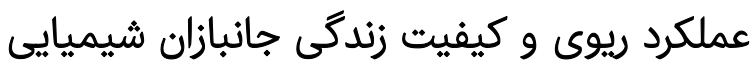

غلامعلى قاسمى

كروه آسيبشناسى و حركات اصلاتى اصلاح، دانشكده علوم ورزشى، دانشكاه اصفهان،

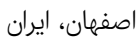

MSc يروين براتىركعتى إيرن

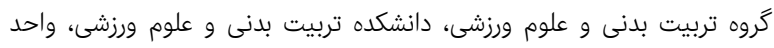

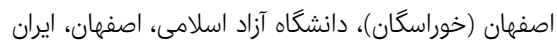

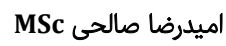

كروه تربيت بدنى و علوم ورزشى، دانشكده علوم انسانى و اجتماعى، دانشكاه

كردستان، سنندج، ايران

اهداف: مصدوميت با عوامل شيميايى ابعاد جسمى، روانى، اجتماعى و معنوى

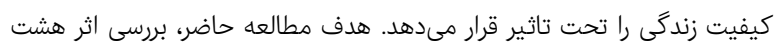

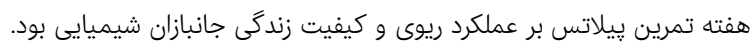

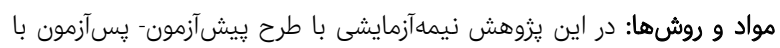

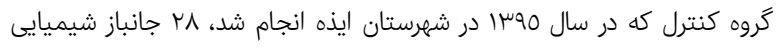

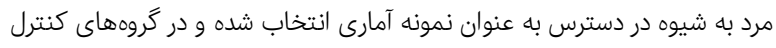

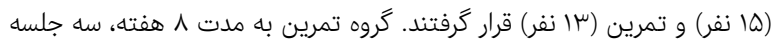

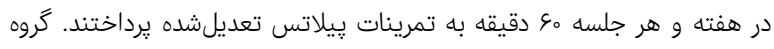

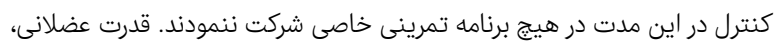

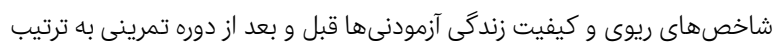

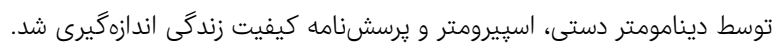

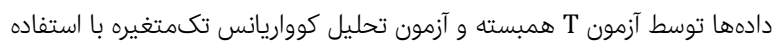

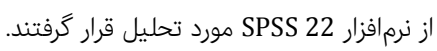

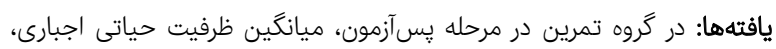

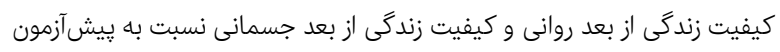

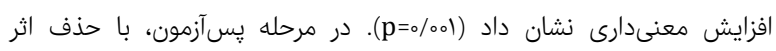

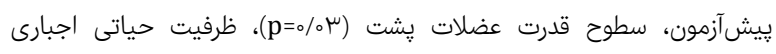
(p=o/\%)

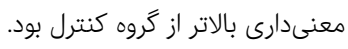

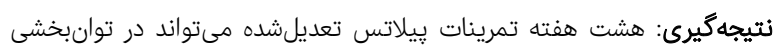

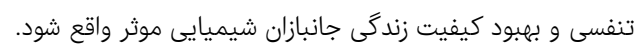

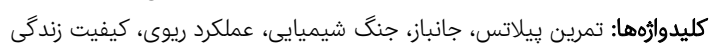

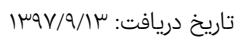

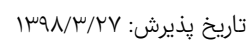

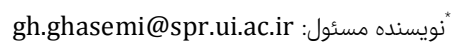

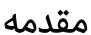

امروزه استفاده از سلاحهاى شيميايى در سطح بينالمللى بهعنوان

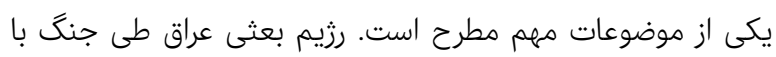

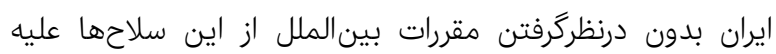

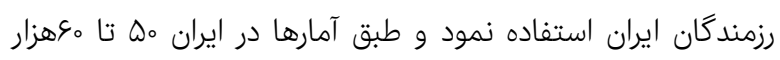

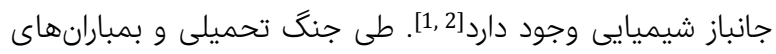

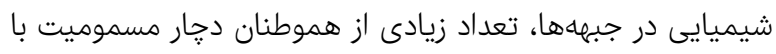

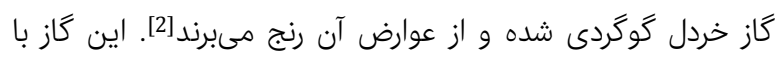




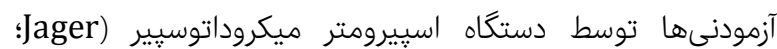
آلمان) و قدرت عضلات يشت با استفاده از دينامومتر ايزومتريكى

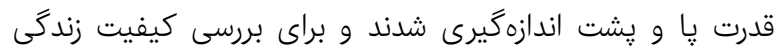

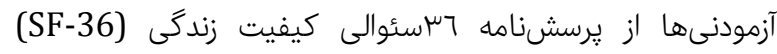
استفاده شد. شيوه اندازهيرى عملكرد ريوى: براى اندازهگيرى عملكرد ريوى

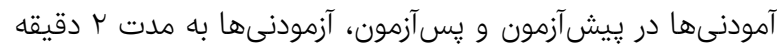

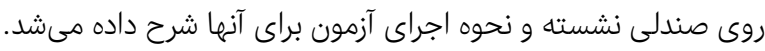

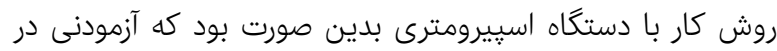

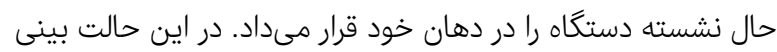

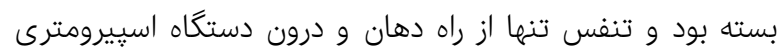

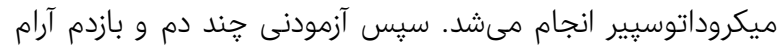

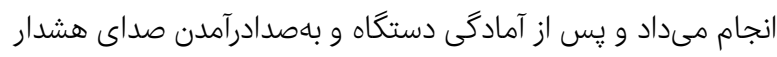

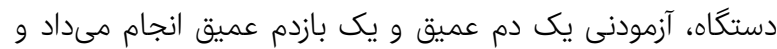

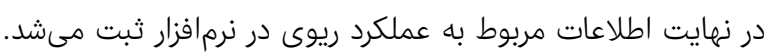
اين نكته قابل ذكر است كه اين دستگاه توسط آقاى سيبل در سال rood در اسيانيا براى تحقيقات خانكى و بالينى ساخته شد.

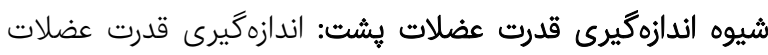

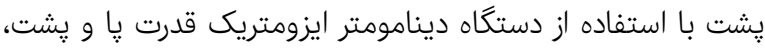

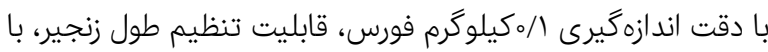

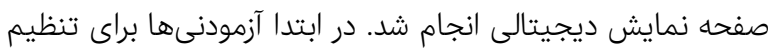

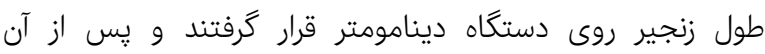

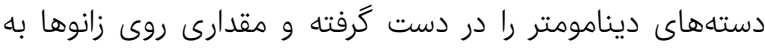

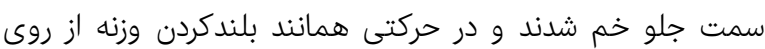
زمين، دستههاى تنسيومتر را با حداكثر قدرت به سمت بـت بالا كشيدند

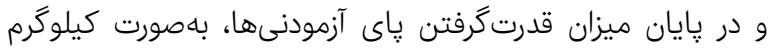
ثبت شد.

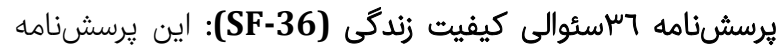

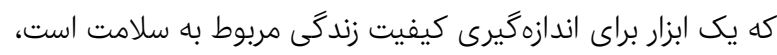

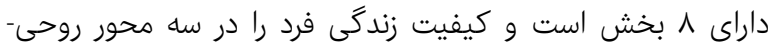

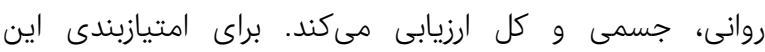

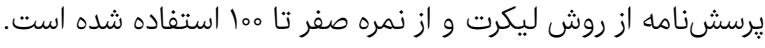

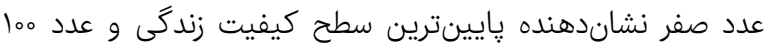

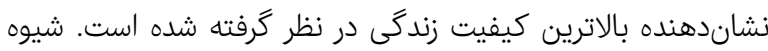

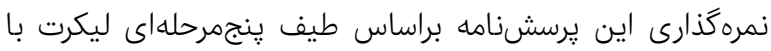

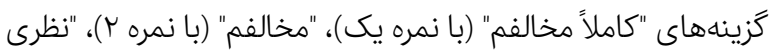

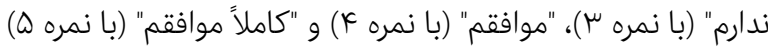

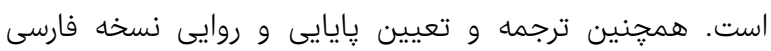

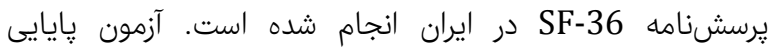

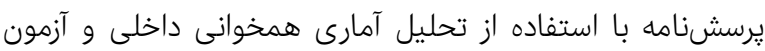

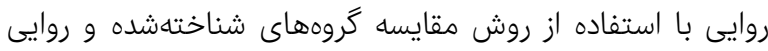

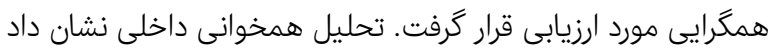
كه مقياسهاى نسخه فارسى SF-36 از حداقل ضرايب استاندارد فردار

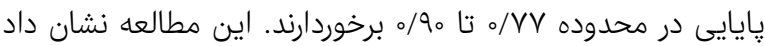

باعث افزايش قدرت عضلات تنفسى و ضخامت عضلات شكمى شد، ولى تغيير معنىدارى در اندازه ظرفيت حياتى اجبارى و هواى

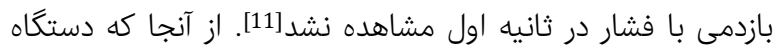

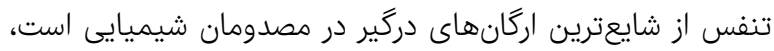

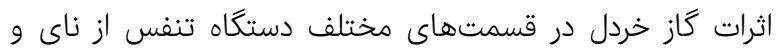
برونشهاى اصلى تا برونشيولهاى تنفسى را تحت درد تاثير قرار

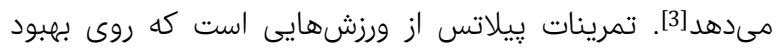
انعطافيذيرى و قدرت در تمام اندامهاى بدن تمركز دارد بدون اين

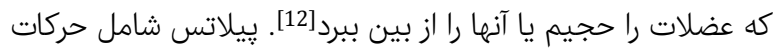
قدرتى و كششى عضلانى است كه شدت تنفس را بالا مىبرد[13].

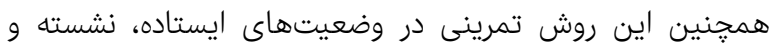

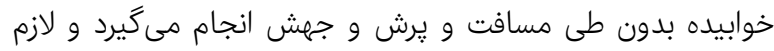

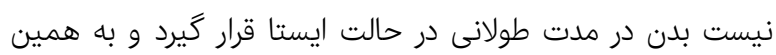

دليل براى معلولان تنفسى مناسب به نظر مى دآيد [11].

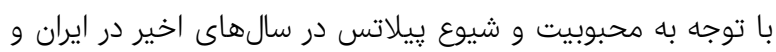

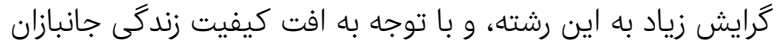

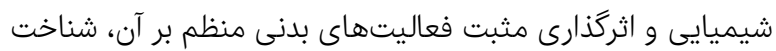

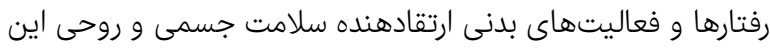

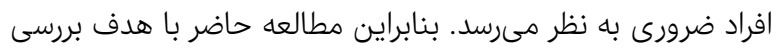

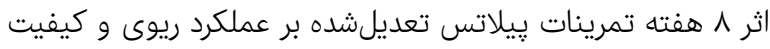

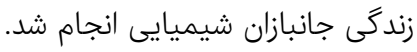

\section{مواد و روشها}

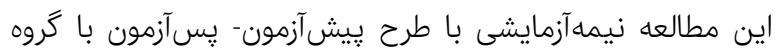

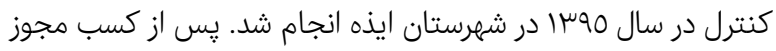

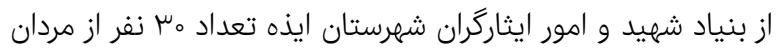

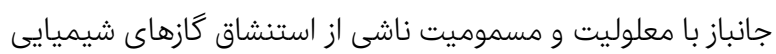
در جنگ تحميلى و بدون بيمارىهايى از قبيل ديابت، فشار خون،

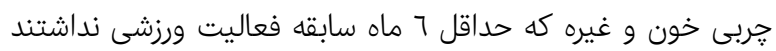

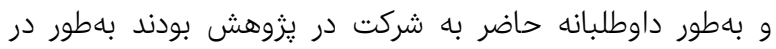

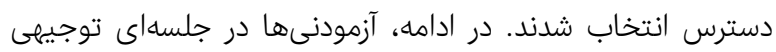

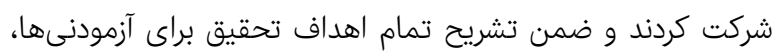

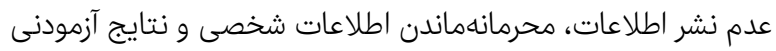
توسط محقق مورد تاييد قرار گرفت.

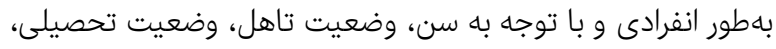

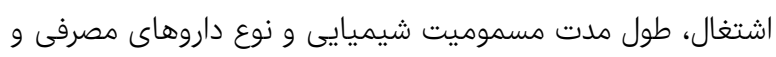

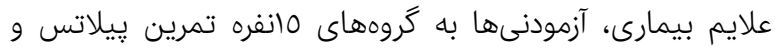

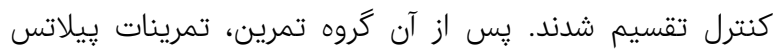

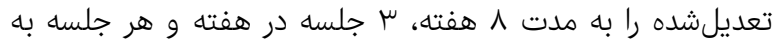

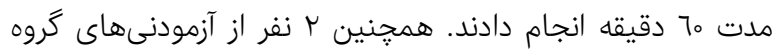

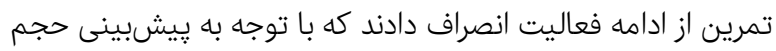

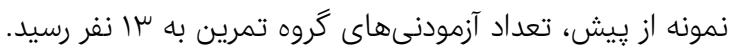

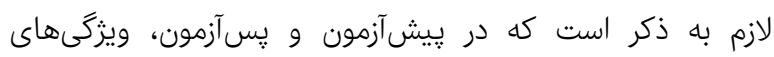

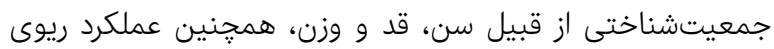


تحليل استنباطى يافتههاى تحقيق و بررسى تغييرات يسآزمون

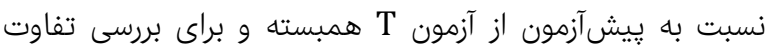

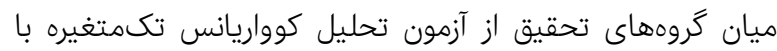
استفاده از نرمافزار SPS 22 SPS استفاده شد.

\section{يافتهها} آزمودنىهاى بزّوهش شامل یץ نفر بودند كه از اين تعداد سا نفر در

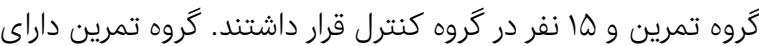

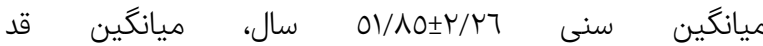

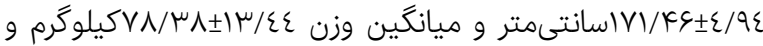

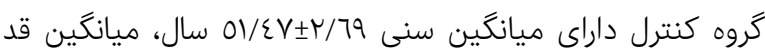

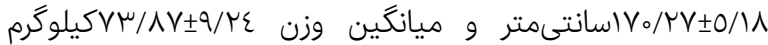
ميانكين متغيرهاى قدرت عضلات يشت، ظرفيت حياتى اجبارى،

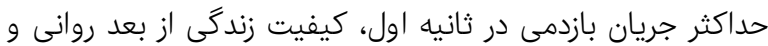

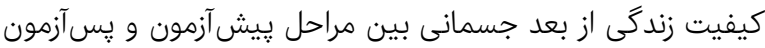

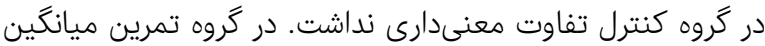
قدرت عضلات بشت و حداكثر جريان بازدمى در ثانيه اول در مر مرحله

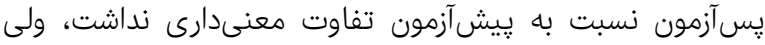

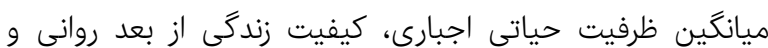

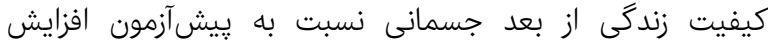

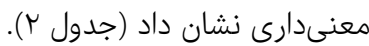

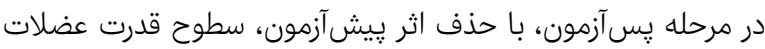

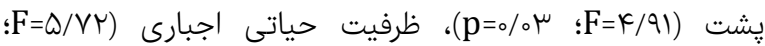

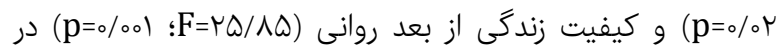

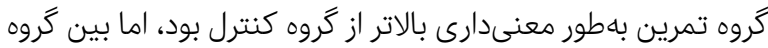

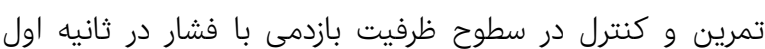

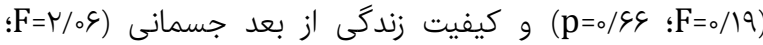
تفاوت معنىدارى وجود نداشت.
نسخه فارسى ابزار استاندارد SF-36 بهمنظور اندازمكيرى كيفيت

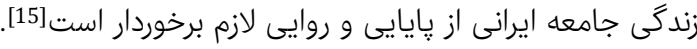

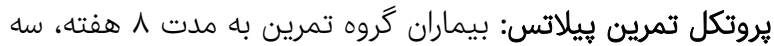

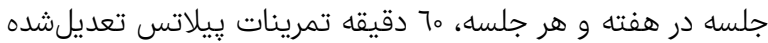

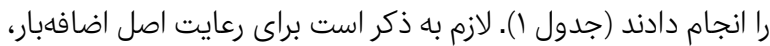

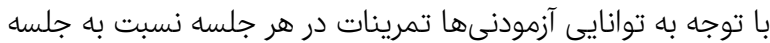

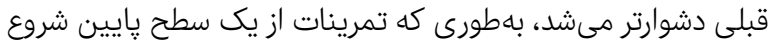

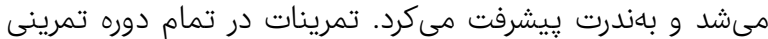

توسط مربى مجرب بِيلاتس و در حضور يرستار انجام مى شئ [8].

\begin{tabular}{|c|c|c|}
\hline زمان & حركات & هفتهها \\
\hline r دقيقه & 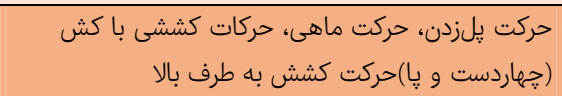 & تمته اول \\
\hline Fo دقيقه & 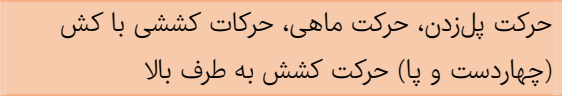 & دوم هفته \\
\hline FQ دقيقه & 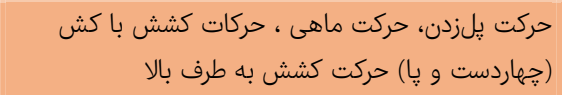 & هفته \\
\hline A دقيقه & 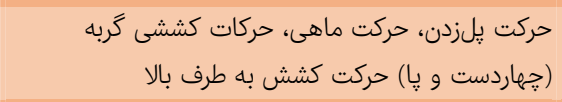 & هفته \\
\hline FA دقيقه & 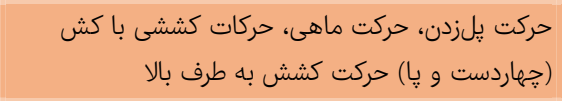 & هفتجم \\
\hline ه دقيقه & 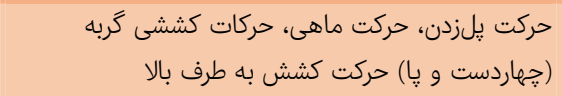 & هفته \\
\hline ه دقيقه & 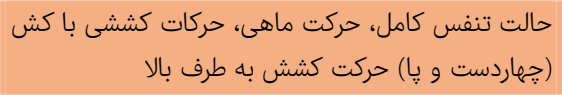 & هفتم \\
\hline هـ دقيقه & 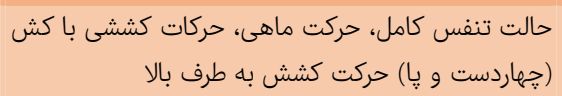 & هفته \\
\hline
\end{tabular}

بامنظور تجزيه و تحليل يافتههاى تحقيق در بخش آمار توصيفى از از إنيان

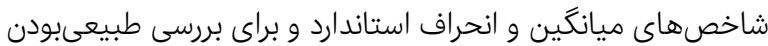
توزيع دادهها از آزمون شإِيرو- ويلك استفاده شد. براى تجزيه و

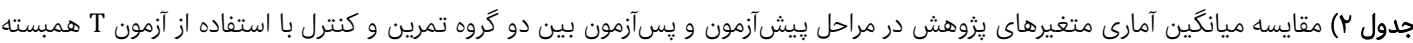

\begin{tabular}{|c|c|c|c|c|c|}
\hline سطح معنىدارى & df & 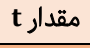 & يس آزمون & 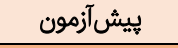 & متغيرها \\
\hline & & & & & قدرت عضلات يشت \\
\hline$\circ / 9 \Delta$ & if & $\circ / F Q$ & $\Delta r / Y Y_{ \pm}+r_{0} / V Y$ & $\Delta r / \varepsilon_{0} \pm 19 / \wedge \mu$ & كروه كنترل \\
\hline$\% \Delta \&$ & ir & $-r / 11$ & $\Delta \Delta / \mu \mu_{ \pm}+r_{0} / V \mu$ & $\kappa Q / \mu \Lambda \pm M I / \Lambda \Lambda$ & كروه تمرين \\
\hline & & & & & ظرفيت حياتى اجبارى \\
\hline$\circ / 14$ & if & $1 / 9 \mu$ & 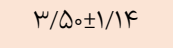 & $\mu / \Delta \mu \pm 1 / \Lambda Q$ & كروه كنترل \\
\hline$\% 001$ & ir & $-K / T 1$ & $\varepsilon / \mu_{\circ} \pm r / \Lambda_{0}$ & $\kappa / \circ \bowtie \pm I / \wedge \varphi$ & كروه تمرين \\
\hline & & & & \multicolumn{2}{|c|}{ حداكثر جريان بازدمى با فشار در ثانيه اول } \\
\hline$\% 9$ & If & l/Ar & $r / ৭ ૬ \pm I / / 。$ & $\mu / \circ \Delta \pm 1 / 19$ & كروه كنترل \\
\hline$\circ / Q 1$ & ir & ०/98 & $\mu / \Upsilon \Lambda \pm 0 / \varphi q$ & $\mu / \Delta \mu \pm 1 / \omega_{0}$ & كروه تمرين \\
\hline & & & & \multicolumn{2}{|c|}{ كيفيت زندگى از بعد روانى } \\
\hline$\circ / V Y$ & If & \& & $\mid K \varepsilon / \varepsilon_{0} \pm G K / K \mu$ & $\mid K N / \Delta_{0} \pm \varepsilon K / K \mu$ & كروه كنترل \\
\hline$\% 001$ & ir & $-9 / 90$ & $1 \Lambda \Delta / T \Psi \pm K \varepsilon / Q \Delta$ & $\wedge r / V \varepsilon_{ \pm} \in \Delta / \circ \mid$ & كَروه تمرين \\
\hline & & & & \multicolumn{2}{|c|}{ كيفيت زندگى از بعد جسمانى } \\
\hline.$/ 91$ & If & $\circ / 1$ & $|\mu \mu / \circ \pm \pm \Lambda| / \Lambda \mu$ & $\mid \Psi \Psi / G 9 \pm \Lambda N / \circ \mu$ & كروه كنترل \\
\hline$\%$ & ir & $-\varphi / \mu r$ & $|S V / \& q \pm F| / \Delta T$ & $\Lambda Y / R \varepsilon_{ \pm} Y Q / K_{0}$ & گروه تمرين \\
\hline
\end{tabular}




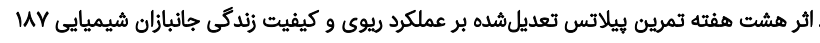

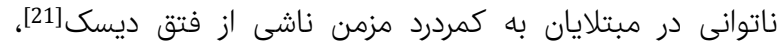
افزايش قدرت عضلانى، تعادل ايستا، دامنه حركتى، تعادل و و

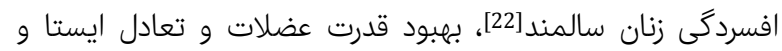

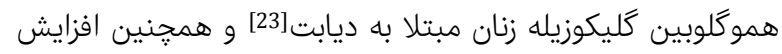

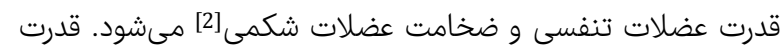

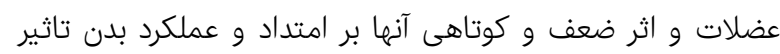

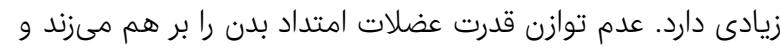

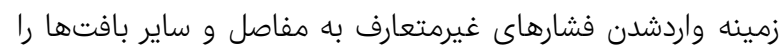

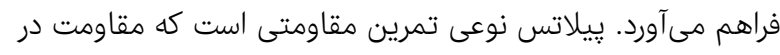

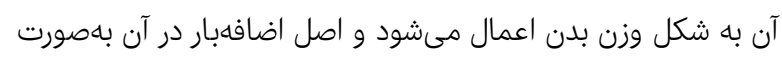

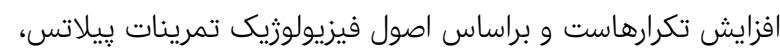
عضلات اسكلتى مىتوانند بهشدت تحت تاثير تمرينات قرار بكيرند.

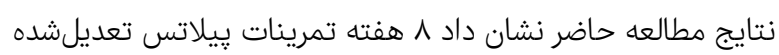

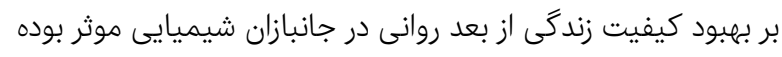

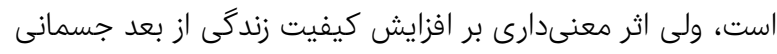

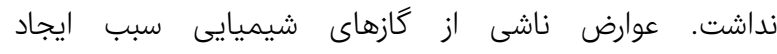

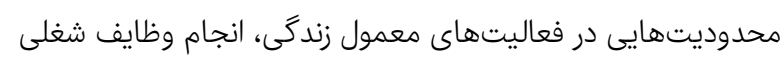
و مشكلات روحى به سبب تغييرات جسمى شده و در مواردى نيز روابط اجتماعى آسيبديدكان را در مقايسه با مردم عادى محدود تغيرات

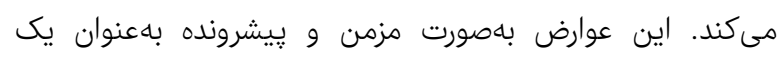

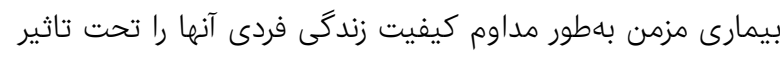

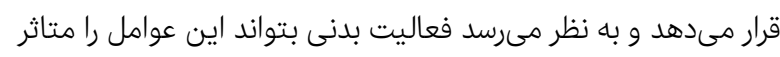

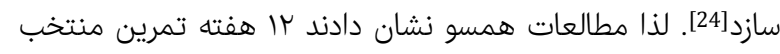

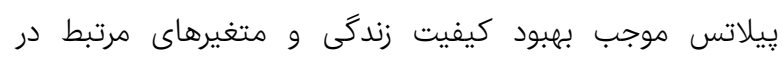

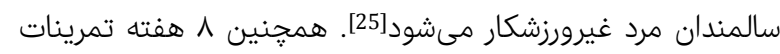

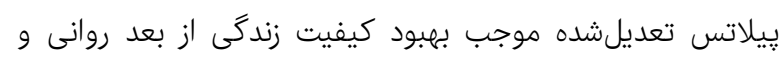

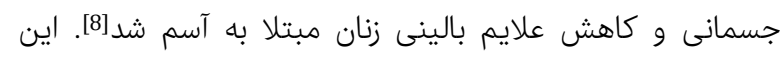

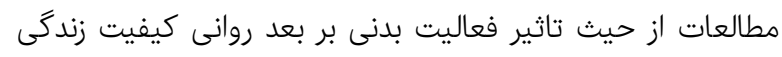

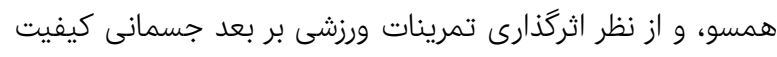

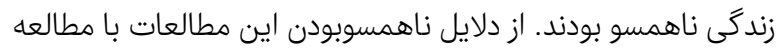
حاضر مىتوان به تفاوت در جامعه آمارى و نوع تمرينات اشاره كرد.

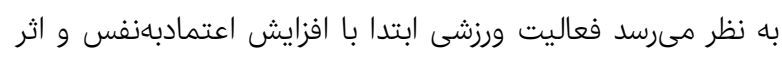

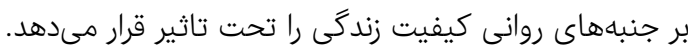

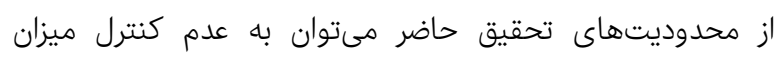

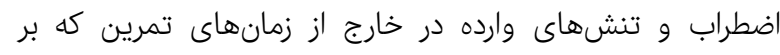
متغيرهاى تحقيق حاضر اثركذار هستند اشاره كرد. بنابراين يِيشنهاد

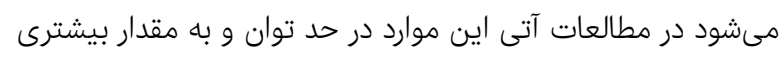

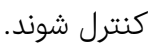

\section{نتيجه گيرى}

هشت هفته تمرينات ييلاتس تعديلشده مىتواند در توانبخشى لئى

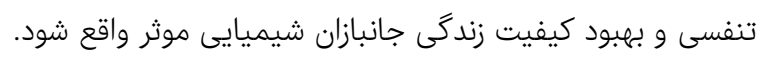
تشكر و قدردانى: نويسندكان اين مقاله بدين وسيله مراتب تشكر و

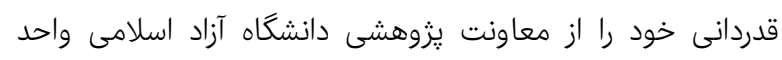

نتايج مطالعه حاضر نشان داد سطوح قدرت عضلات يشت و ظرفيت

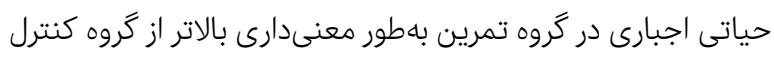

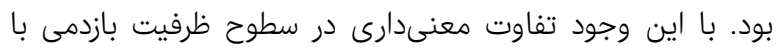

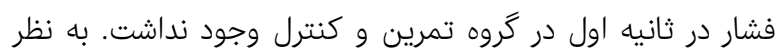

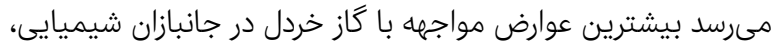

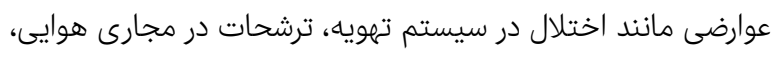
خونريزى مجارى هوايى، يروفيوزن، اختلالات بيوشيميايى ريه،

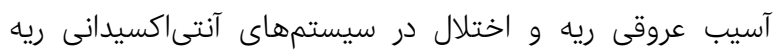

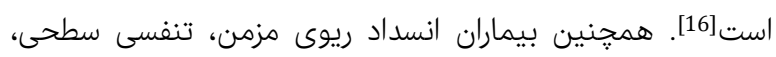

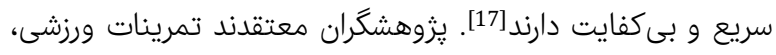

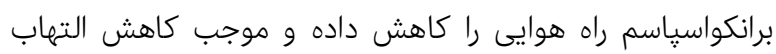

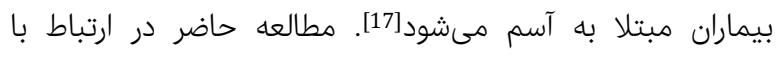
تغييرات علايم بالينى ناشى از بهبود قدرت عضلات تنفسى و و

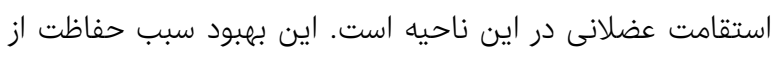

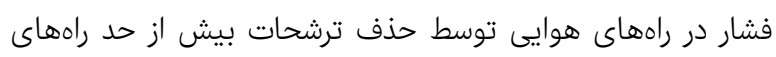

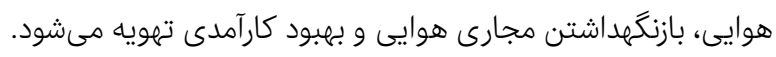

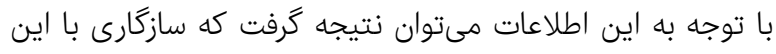

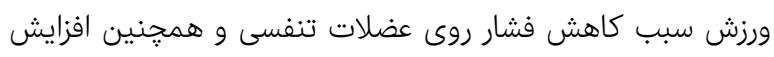
كارآيى اين عضلات، كاهش مقاومت در برابر دم و بازدم و بهبود

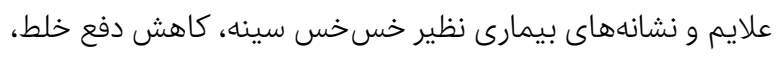

كاهش احساس كوتاهى نفس و كاهش سرفه مىشود [8].

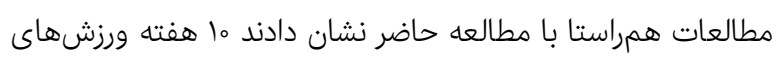

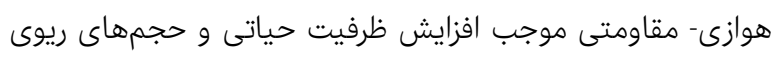

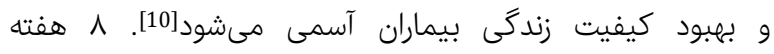

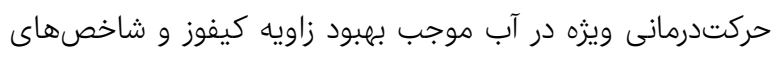

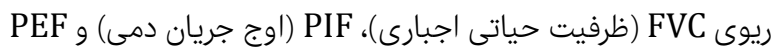

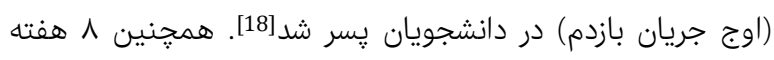

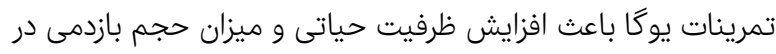

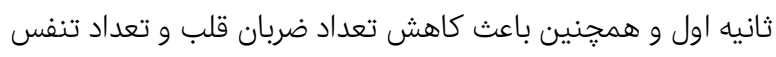

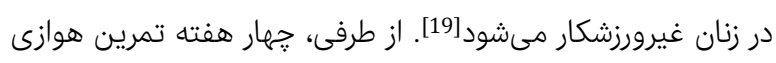

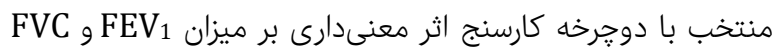

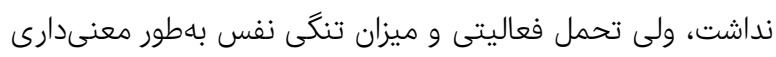

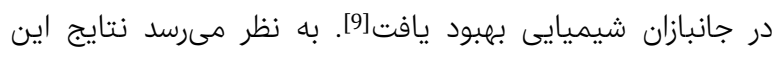

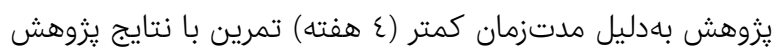

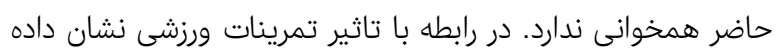

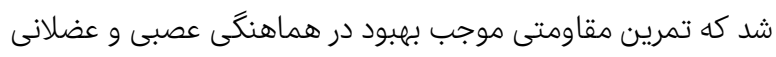
و افزايش حجم عضلانى مىشود، در نتيجه سطح مقطع با توليد

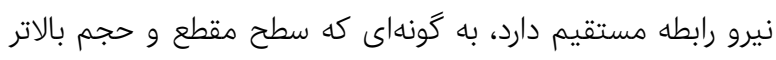

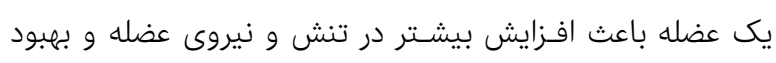
توان هوازى مىشود [20].

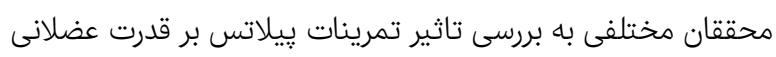

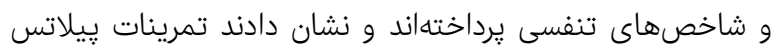
باعث افزايش معنىدار قدرت عضلات شكم و پشت و و كاهش ميزان 
Med J. 2010;68(6):348-54. [Persian]

11- Giacomini MB, da Silva AM, Weber LM, Monteiro MB.

The Pilates method increases respiratory muscle strength and performance as well as abdominal muscle thickness. J Bodyw Mov Ther. 2016;20(2):258-64.

12- de Alvarenga GM, Charkovski SA, dos Santos LK, da Silva MAB, Tomaz GO, Gamba HR. The influence of inspiratory muscle training combined with the Pilates method on lung function in elderly women: a randomized controlled trial. Clinics. 2018;73:e356.

13- Rydeard R, Leger A, Smith D. Pilates-based therapeutic exercise: effect on subjects with nonspecific chronic low back pain and functional disability: a randomized controlled trial. J Orthop Sports Phys Ther. 2006;36(7):472-84.

14- Wong T. Pilates and the stroke patient. Balanced Body Pilates COREterly. 2010.

15- Jafari F, Gitynavard F, Soroush MR, Mousavi B. Quality of life in chemical war victims with sever pulmonary damage. Iran J War Public Health. 2012;4(1):46-52. [Persian]

16- Kavei B, Fagihzadeh S, Eskandari F, Kazemnejad A, Gazanfari T, Soroush MR. Studying the surrogate validity of respiratory indexes in predicting the respiratory illnesses in wounded people exposed to sulfur mustard. J Arak Univ Med Sci . 2011;13(4):75-82. [Persian]

17- Zakerimoghadam M, Shaban M, Kazemnejad A, Tavasoli K. The effect of breathing exercises on fatigue level of COPD patients. J Hayat. 2006;12(3):17-25. [Persian]

18- Azizi A, Mahdavinejad R, Taheri Tizabi A, Jafarnejad T. The effect of 8 weeks specific aquatic therapy on kyphosis angle and some pulmonary indices in male university students with kyphosis. J Kerman Univ Med Sci. 2012;19(5):440-50. [Persian]

19- Sharifi G, Tabatabaei SH, Babaei A, Tollabi M. The effect of 8 weeks yoga training on respiratory function and heart of non-athlete females. Toloo-e Behdasht. 2015;14(2):69-78. [Persian]

20- Negaresh R, Ranjbar R, Habibi A, Gharibvand MM. The effects of eight weeks of resistance training on some muscle hypertrophy and physiological parameters in elderly men. J Geriatr Nurs. 2016;3(1):62-75. [Persian]

21- Shahrokhi M, Balouchi R, Ebrahimi H. Effect of six week Pilates exercises on disability, abdominal and back muscle strength in patients with chronic low back pain due to disc herniation. Stud Sport Med. 2015;7(17):98-83. [Persian]

22- Kamali A, Mahdavinezhad R, Norouzi K. The effect of selected Pilates exercises on thigh muscle strength and depression in elderly women. J Paramed Sci Rehabil. 2016;5(2):67-75. [Persian]

23- Ebrahimi F, Mahdavinejad R, Jalili HR. The effects of selected Pilates exercises on muscle strength, balance and HbA1c in female patients with diabetes type 2. J Sport Med. 2015;7(2):251-65. [Persian]

24- Abbasi M, Jamali HA, Omidi Oskouei A. Quality of life of chemically-disabled war veterans involved in pulmonary complications of sulfur mustard gas in Sardasht. Qom Univ Med Sci J. 2012;5(4):34-9. [Persian] 25- Bahram ME, Pourvaghar MJ, Sadeh MR. Effect of 12 weeks of chosen Pilates exercise on the quality of life of healthy nonathletic people. Toloo-e Behdasht. 2014;13(1):93-103. [Persian]

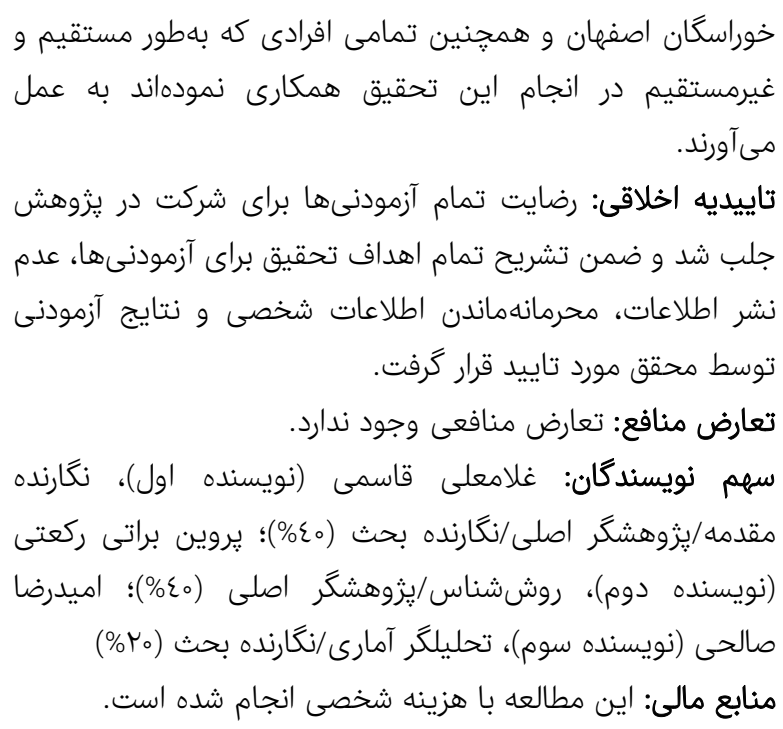

1- Ayoubi F, Ghazanfari T, Askari N, Naghizadeh MM, Soroush MR. Serum level of SDF-1 $\alpha$ (CXCL12) in chemical victims with respiratory complications. Iran J War Public Health. 2014;6(2):55-63. [Persian]

2- Nasiripoor Z, Abedi B, Hajirasouli M. Effect of a training program on quality of life of severe respiratory chemical veterans; a case study of Markazi province. Iran J War Public Health. 2016;8(2):89-94. [Persian]

3- Abedi A, Koohestani H, Roosta Z. The short-term effect of chest physiotherapy on spirometric indices in chemical warfare victims exposed to mustard gas. Armaghan-e Danesh. 2008;13(3):71-81. [Persian]

4- Balali-Mood M, Hefazi M. A review on the delayed complications of sulphur mustard poisoning. J Birjand Univ Med Sci. 2005;12(3 and 4):9-15. [Persian]

5- Asai K, Kanazawa H, Kamoi H, Shiraishi S, Hirata K, Yoshkawa J. Increased levels of vascular endothelial growth factor in induced sputum in asthmatic patients. Clin Exp Allergy. 2003;33(5):595-0.

6- Meamari H, Koushkie Jahromi M, Fallahi AA, Sheikholeslami R. Influence of structural corrective and respiratory exercises on cardiorespiratory indices of male children afflicted with kyphosis. J Rehabil. 2017;18(1):5162. [Persian]

7- Mirbagher-Ajorpaz N, Rezaei M. The effect of pulmonary rehabilitation program on quality of life of elderly patients with chronic obstructive pulmonary disease. Zahedan J Res Med Sci. 2011;13(1):30-5. [Persian]

8- Mirzakhanian M, Ghasemi G, Sadeghi M, Ghasemi R. The effects of modified Pilates training on quality of life and clinical symptoms in female asthmatic patients. J Res Sport Rehabil. 2015;3(5):43-50. [Persian]

9- Tari M, Fallah Mohammadi Z, Dabidi Roshan V, Oliaei $M$. The effect of a selected aerobic exercise course on the rate of FEV1 and FVC, activity tolerance, and dyspnea in chemical pulmonary veterans. Olympic J. 2009;17(1):1932. [Persian]

10- Fesharaki M, Paknejad SMJO, Kordi R. The effects of aerobic and strength exercises on pulmonary function tests and quality of life in asthmatic patients. Tehran Univ 